\title{
Prevalence of Scapular Dyskinesia in Young Adults with Trapezitis - A Cross-Sectional Study
}

\author{
Jainy P Patel ${ }^{1}$, Alpa Purohit ${ }^{2}$ \\ ${ }^{1}$ Postgraduate Physiotherapy Student, Ahmedabad Institute of Medical Sciences (AIMS) affiliated to Gujarat \\ University, Ahmedabad, Gujarat, India \\ ${ }^{2}$ Senior Lecturer and Vice Principal, Ahmedabad Institute of Medical Sciences, Ahmedabad, Gujarat, India
}

Corresponding Author: Jainy P Patel

\begin{abstract}
Background: An abnormal movement of the scapula during shoulder movement is termed as scapular dyskinesia and is an often-forgotten cause of pain and dysfunction. The scapula is a key part of the upper limb kinematic chain and is a vital component of the glenohumeral rhythm. Trapezitis is an inflammatory pain resulting from trapezius muscle. Trapezius is a stabilizer in Scapular dynamics contributing to scapulohumeral rhythm. Weakness or improper activation of Scapular stabilizers can alter Scapular positioning and mechanics. Aim of this study is to find prevalence of Scapular Dyskinesia in young adults with Trapezitis.

Methodology: One hundred eighteen young adults of age 18-25 years with Trapezitis selected by convenience sampling participated in this cross sectional study. Participants were allotted to three groups (mild, moderate, severe) according to severity of Trapezitis on the basis of their VAS score. Dynamic scapular Dyskinesis test was used to assess Scapular Dyskinesia. Statistical analysis was done using Microsoft excel version 2010.

Results: 118 participants had participated in the study. Out of which $32 \%$ participants had mild trapezitis, $37 \%$ had moderate trapezitis and $31 \%$ had severe trapezitis. In subjects with mild trapezitis $37 \%$ of their population had scapular dyskinesia whereas in subjects with moderate trapezitis $57 \%$ had scapular dyskinesia and in participants with severe trapezitis, scapular dyskinesia was witnessed in $72 \%$ of their population.
\end{abstract}

Conclusion: Present study evidences high prevalence of Scapular Dyskinesia in young adults with Trapezitis.

Key Words: Scapular Dyskinesia, Trapezitis, Young adults

\section{INTRODUCTION}

The scapula plays a crucial role in coordinating and maintaining complex shoulder kinematics ${ }^{[1]}$. Scapular Dyskinesia is an alteration in the normal position or motion of scapula during coupled scapulohumeral movements."Dys" alluded to 'alteration of' while "kinesis" alludes to 'movement' that demonstrates the loss of ordinary control of Scapular movement ${ }^{[3]}$. Inflexibility and contractures of muscles and ligaments around shoulder can affect the position and movement of scapula ${ }^{[6]}$
The scapula is anatomically and biomechanically intimately involved in shoulder function ${ }^{[6]}$. Scapula provides synchronous scapular rotation during humeral motion and function as a link in kinetic chain ${ }^{[7]}$. If Scapular position is impaired the forces generated from lower extremity and trunk will not be effectively transmitted to upper extremity. This loss of link function results in altered scapular biomechanics ${ }^{[6]}$. Serial muscular activation patterns stabilizes scapula and increases the control of its motion and position as the arm is moved ${ }^{[6]}$. Scapular stabilization requires 
coupling of upper and lower trapezius, serratus anterior and rhomboids muscles ${ }^{[6]}$. Inhibition or disorganization of activation patterns in Scapular stabilizing muscles can alter the scapular position thus causing scapular Dyskinesia ${ }^{[6]}$. Altered Scapular orientation is proven a risk factor for many shoulder and neck pathologies such as Impingement and neck pain ${ }^{[2]}$.

Trapezius is an extensive muscle subdivided into upper middle and lower parts ${ }^{[4]}$. It extends longitudinally from occipital bone to lower thoracic vertebra and laterally to spine of scapula ${ }^{[5]}$. This muscle is a dominant stabilizer of scapula normally operating synergistically with other scapular muscles, mostly serratus anterior ${ }^{[4]}$. It also contributes to scapulohumeral rhythm through attachment on clavicle and scapula ${ }^{[5]}$. Trapezius and serratus anterior acts synergistically to produce many actions of scapula or clavicle typically associated with flexion or abduction of scapula ${ }^{[4]}$.

The upper trapezius muscle is designated as postural muscle and it is highly susceptible to overuse ${ }^{[8]}$. Trapezitis defined as inflammation of trapezius muscle ${ }^{[8]}$. It is usually caused by placing too much stress or strain over trapezius muscle ${ }^{[9]}$. Trapezitis is most common musculoskeletal disorder ${ }^{[9]}$. It is common in age group of 18-30 years. Altered activation, poor control or reduced strength of different parts of trapezius muscle has been linked with abnormal Scapular movements, often associated with pain ${ }^{[4]}$. Abnormal posture makes the area vulnerable to a range of musculoskeletal Conditions by imparting large stresses to the neck and shoulders, as well as weakening the soft tissues by decreasing the biomechanical functions of the area, and inducing muscle stiffness and hypokinetics ${ }^{[10]}$

Previous studies have supported the stabilizing role of trapezius muscle on scapular motion and also its contribution to scapulohumeral rhythm. However, the direct association of trapezitis with scapular dyskinesia has not yet been established. Therefore, as study on prevalence of
Scapular Dyskinesia has not been conducted and explored much,. The main aim of this study is to know the prevalence of Scapular Dyskinesia in young adults with Trapezitis.

\section{METHOD}

An observational study was conducted from February 2021 to June 2021. One hundred eighteen healthy young adults were selected by convenience sampling. Data for the study conducted was collected from various colleges and community areas of Ahmedabad and Himmatnagar. The subjects were allotted to three groups namely, mild moderate and severe according to severity of trapezitis based on their score on VAS scale. Scapular Dyskinesia was assessed using Dynamic Scapular Dyskinesis test.

Inclusion Criteria Willingness of subject to participate, both male and female, Age (18-25years), Subjects with unilateral trapezitis.

Exclusion Criteria Cervical spine disorders, Any Upper limb or vertebral fracture; Any head injury; Neurological disorder

\section{OUTCOME MEASURES}

\section{Dynamic Scapular Dyskinesis Test}

This consists of weighted shoulder flexion and abduction movement while the scapular motion is closely observed. The patient holds a 2-pound (1 kg) weight in each hand and first forward flexes and then abducts both arms. The clinician observes whether there is any winging, or prominence of the medial or inferior scapular borders, or any lack of coordinated movements (such as early scapular elevation or shrugging, when lifting the arm, or fast downward rotation when lowering the arm). Any deviation from the norm is noted as a 'yes' (dyskinesis is present) or 'no'.

\section{Visual Analogue Scale}

VAS was used to evaluate and quantify the pain experienced by the subjects. Origin of the scale is indicated as "NO PAIN" and the terminal end as 
"MOST SEVERE PAIN". The patient was instructed to move the indicator to represent his/her pain perceived. At the back of the scale 0 to 10 numerical with a distance of $1 \mathrm{~cm}$ between them were marked.

\section{RESULTS}
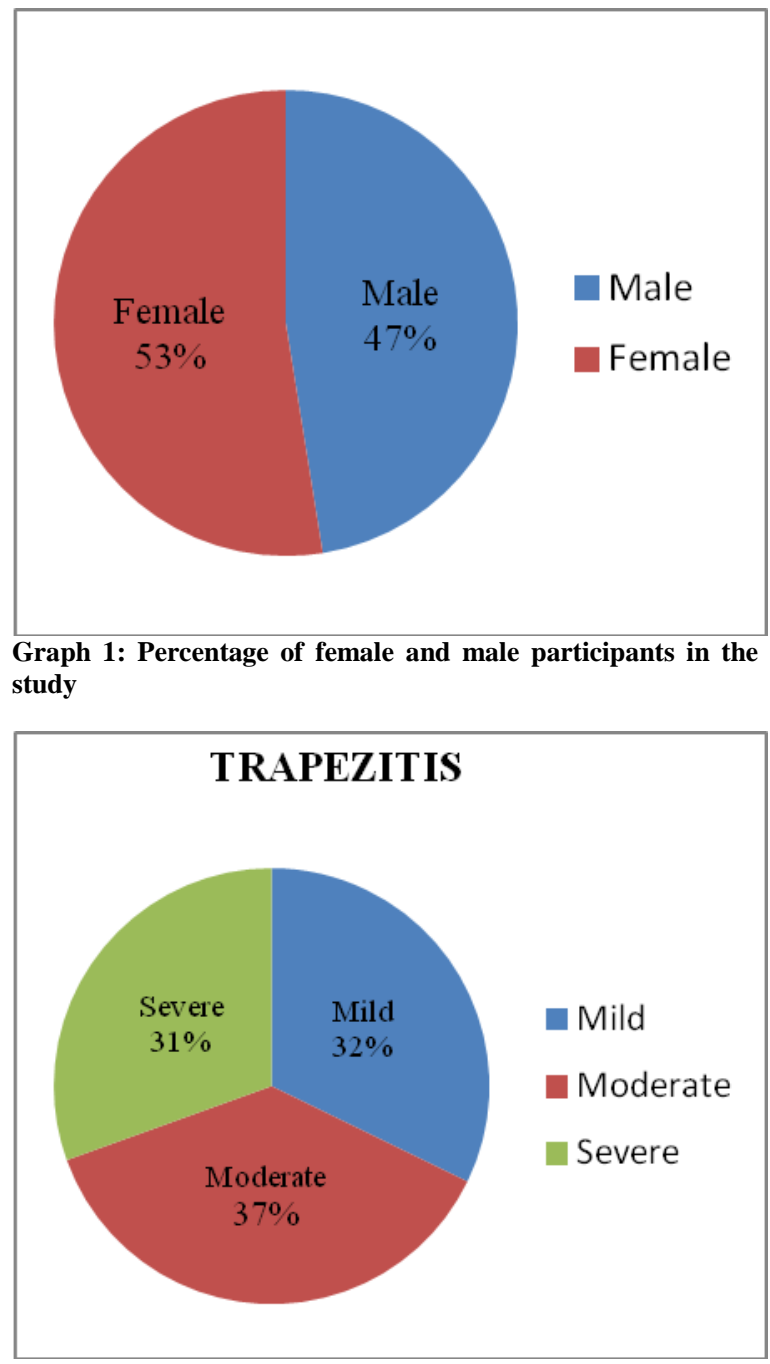

Graph 2 : Percentage of participants according to the severity of trapezitis

Statistical analysis was done using Microsoft Excel 2010. One hundred eighteen subjects participated in the study. Out of 118 participants, 56 were boys and 62 were girls which constituted $47 \%$ and $53 \%$ respectively. Mean age of the subjects was 22.5 years. Graph 1 illustrates the percentage of male and female participants prevailing in this study. Graph 2 demonstrates the population which falls into three categories namely mild moderate and severe on the basis of visual analogue score of trapezitis. Graph 3,4 and 5 evidences the percentage of participants having scapular dyskinesia on the basis of severity of trapezitis.

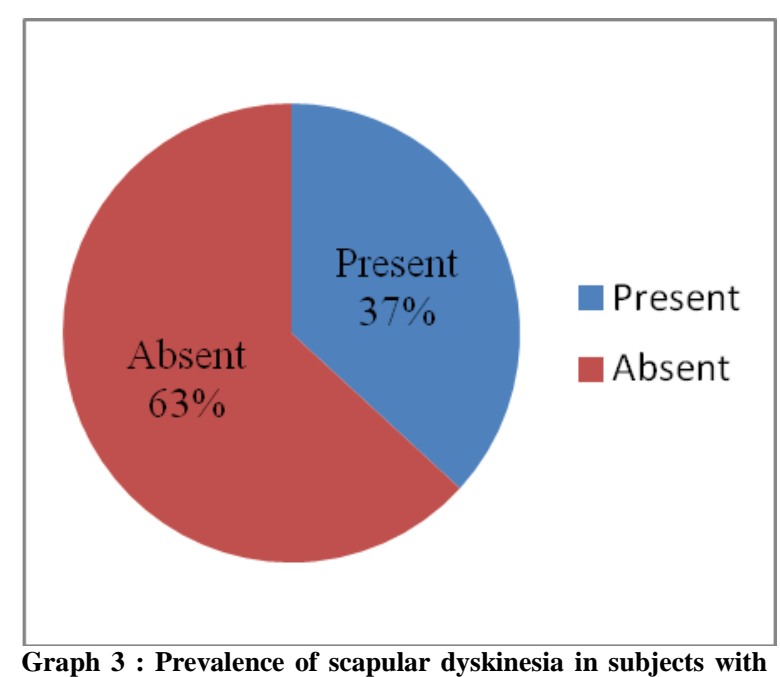
mild trapezitis

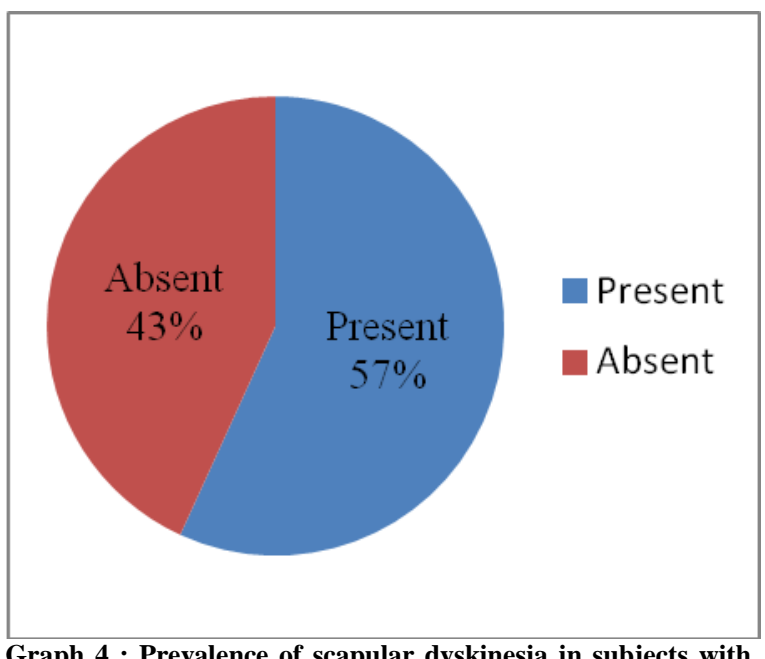
moderate trapezitis

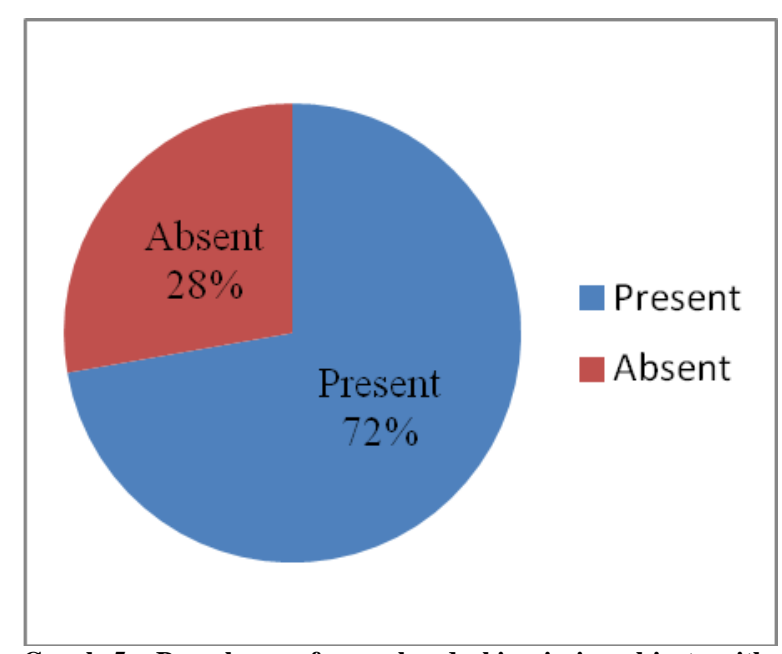

Graph 5 : Prevalence of scapular dyskinesia in subjects with severe trapezitis 
Result suggests $32 \%$ participants were having mild trapezitis out of which scapular dyskinesia was present in $37 \%$ of them. Also, $37 \%$ subjects were having moderate trapezitis. Among them 57\% had scapular dyskinesia. High prevalence of scapular dyskinesia was seen $72 \%$ of population who were having severe trapezitis. (31\%)

\section{DISCUSSION}

Present study was conducted on 118 subjects with Trapezitis to find out the prevalence of Scapular Dyskinesia in them. Result of the present study shows significantly high prevalence of Scapular Dyskinesia in young adults with Trapezitis

Result shows that, in subjects with mild trapezitis $37 \%$ of their population had scapular dyskinesia whereas in subjects with moderate trapezitis $57 \%$ had scapular dyskinesia and in participants with severe trapezitis, scapular dyskinesia was witnessed in $72 \%$ of their population. This variation in the prevalence of dyskinesia among individuals with trapezitis suggests that severity of trapezitis creates an impact on scapular dynamics and thus can variably alter its position. This might be due to difference in rate of upper trapezius activation in trapezitis. Upper trapezius could be more hyperactive and increased its pull in severe trapezitis compared to mild and moderate trapezitis and thereby alternating scapular mechanics in scapulohumeral rhythm.

Impaired scapular kinematics and associated muscle activation are thought to result in shoulder disorders such as pain, restricted range of motion, and functional disability. Based on these assumptions, researchers have identified insufficient posterior tipping, external rotation, and upward rotation; decreased serratus anterior and lower trapezius activity; and increased upper trapezius muscle activity in patients with shoulder impingement ${ }^{\text {[2] }}$

Research has highlighted that the upper and lower trapezius along with serratus anterior are the muscles that mostly affect scapular movement and cause dyskinesia. When the scapula biomechanics are considered in relation to the anatomy, it becomes evident that the combination of movements, planes and muscles involved there is a vast array of combinations that could lead to abnormal movement function [11]

Studies suggest that trapezius and the serratus anterior muscles have been linked to the development of dyskinesis in both shoulder impingement and shoulder instability. In impingement, the upper and lower trapezius along with the serratus anterior has altered their activation pattern, with the trapezius showing a greater strength of activation compared to the serratus anterior ${ }^{[12]}$.

Studies have identified that individuals with shoulder pain that individuals with shoulder pain during elevation of the arm often present with abnormal or labored scapula movements in conjunction with (1) excessive activation of the upper trapezius and (2) decreased and/or delayed activation of the lower and middle trapezius and the serratus anterior. Specifically, excessive activation of the upper trapezius is likely associated with increased elevation of the clavicle coupled with undesired anterior tilt of the scapula. ${ }^{[4]}$

Peat and Grahame investigated trapezius, serratus anterior and deltoid EMG in people with and without shoulder pathology. They found that in those with shoulder pathology, UT showed increased activity during arm elevation and lowering (between 40 to 100 degrees of arm elevation). Also, serratus anterior showed decreased activity at some humeral elevation angles (between 70 to 100 degrees) compared to healthy controls. This increase in UT activity has been found in numerous other studies on UT activation and shoulder pathology ${ }^{(13)}$.

Moreover imbalances in force production of the serratus anterior and UT can result in a scapular elevation motion (or early shoulder shrugging). This may cause excess superior translation of the scapula, 
with less efficient upward rotation and reduced posterior tilting ${ }^{[13]}$.

One of the unneglectable limitations of this study is that specific occupation has not been included. In future it is recommended to carry out study on various populations such as computer workers, Teachers, Medical staff and those who are more prone to these outcomes. Also, Postural and other Physiological factors which could alter Scapular dynamics should be taken into consideration in future.

\section{CONCLUSION}

The current study evidences that trapezitis plays significant role in causing Scapular Dyskinesia. Moreover present study suggests high prevalence of Scapular Dyskinesia in young adults with Trapezitis.

\section{ACKNOWLEDGMENTS}

I am very grateful to my guide and my parents who supported me constantly throughout the study. I am also thankful to all the subjects who participated in this study to make it worth.

\section{Conflict of Interest: None}

\section{Source of Funding: None}

\section{Ethical Approval: Approved}

\section{REFERENCES}

1. Lefèvre-Colau MM, Nguyen C, Palazzo C, Srour F, Paris G, Vuillemin V, Poiraudeau S, Roby-Brami A, Roren A. Recent advances in kinematics of the shoulder complex in healthy people. Annals of physical and rehabilitation medicine. 2018 Jan;61(1):56-9

2. Huang TS, Lin JJ, Ou HL, Chen YT. Movement pattern of scapular dyskinesis in symptomatic overhead athletes. Scientific reports. $2017 \mathrm{Jul}$ 26;7(1):1-7.

3. Kibler WB, Ludewig PM, McClure P, Uhl TL, Sciascia A. Scapular Summit 2009: introduction. July 16, 2009, Lexington, Kentucky. The Journal of orthopaedic and sports physical therapy. 2009 Nov 1;39(11):A1-3.

4. Camargo PR, Neumann DA. Kinesiologic considerations for targeting activation of scapulothoracic musclespart 2: trapezius. Brazilian journal of physical therapy. 2019 Nov 1;23(6):46775.

5. Raina BS, Manhas A, Jothilingam M. Comparison of Ultrasound Therapy \& Transcutaneous Electrical Nerve Stimulation in the Treatment of Upper Trapezitis. Annals of the Romanian Society for Cell Biology. 2021 May 17;25(6):1121-31.

6. Kibler BW, McMullen J. Scapular dyskinesis and its relation to shoulder pain. JAAOS-Journal of the American Academy of Orthopaedic Surgeons. 2003 Mar 1;11(2):142-51.

7. Kibler WB, Sciascia A. Current concepts: scapular dyskinesis. British journal of sports medicine. 2010 Apr 1;44(5):300-5.

8. Khare D, Pathak R. Effectiveness of elastic resistance band exercises versus conventional exercises on cases of trapezitis: A comparative study. International Journal of Orthopaedics. 2018;4(1):174-8.

9. Ravish VN, Helen S. To compare the effectiveness of myofascial release technique versus positional release technique with laser in patients with unilateral trapezitis. Journal of Evolution of Medical and Dental Sciences. 2014 Mar 3;3(9):2161-7.

10. Park SH, Lee MM. Effects of Lower Trapezius Strengthening Exercises on Pain, Dysfunction, Posture Alignment, Muscle Thickness and Contraction Rate in Patients with Neck Pain; Randomized Controlled Trial. Medical science monitor: international medical journal of experimental and clinical research. 2020;26:e920208-

11. Panagiotopoulos AC, Crowther IM. Scapular dyskinesia, the forgotten culprit of shoulder pain and how to rehabilitate. Sicot-j. 2019;5. 
12. Lopes AD, Timmons MK, Grover M, Ciconelli RM, Michener LA. Visual scapular dyskinesis: kinematics and muscle activity alterations in patients with subacromial impingement syndrome. Archives of physical medicine and rehabilitation. 2015 Feb 1;96(2):298-306.

13. Chris Mallac. Upper trapezius: the key to scapulohumeral rhythm. In musculoskeletal injuries, neck and back injuries, overuse injuries. Sports injury bulletin.

How to cite this article: Patel JP, Purohit A. Prevalence of scapular dyskinesia in young adults with trapezitis - a cross-sectional study. Int J Health Sci Res. 2021; 11(7):63-68. DOI: https://doi.org/10.52403/ijhsr.20210710 